\title{
Optical Properties of InAs Quantum Dot Array Ensembles with Predetermined Lateral Sizes from 20 to $40 \mathrm{~nm}$
}

\author{
Emanuele Uccelli ${ }^{1,2}$, Laura Waller ${ }^{1,3}$, Max Bichler ${ }^{1}$, Gerhard Abstreiter ${ }^{1}$, and Anna Fontcuberta i Morral ${ }^{1,2 *}$ \\ ${ }^{1}$ Walter Schottky Institute, and Physik Department, Technische Universität München, Am Coulombwall 3, D-85748 Garching, Germany \\ ${ }^{2}$ Laboratoire des Matériaux Semiconducteurs, Institut des Matériaux, Ecole Polytechnique Fédérale de Lausanne, CH-1015 Lausanne, Switzerland \\ ${ }^{3}$ Electrical Engineering and Computer Science, Massachusetts Institute of Technology, 77 Massachusetts Ave, Cambridge, MA 02139, U.S.A. \\ Received March 2, 2009; revised January 23, 2010; accepted January 26, 2010; published online April 20, 2010
}

Cleaved edge overgrowth and selective area epitaxy were combined for the synthesis of InAs quantum dot (QD) arrays with lateral sizes from 20 to $40 \mathrm{~nm}$. The optical properties were locally assessed by confocal photoluminescence spectroscopy experiments at liquid helium temperature. The emission lines redshift as the lateral size of the QDs is increased. In agreement with a narrow size distribution, significantly narrow emission lines are observed for measurements in QD ensembles. Excitation power dependent luminescence measurements were realized on QD ensembles. A shell filling behavior was observed. The same measurements realized on single QDs led to the observation of multiple excitonic effects. Polarization dependent luminescence measurements indicate the existence of in-plane optical anisotropy, which strictly follows in-plane morphological anisotropy of the QDs. These results are encouraging for the use of quantum dot arrays in quantum information science and technology, as well as for new device concepts. (C) 2010 The Japan Society of Applied Physics

\section{Introduction}

Self-assembled quantum dots (QDs) are known to have unique optical and electronic properties, which are mainly derived from their strong carrier confinement, leading to atomic-like discretization of the electronic energy levels. ${ }^{1,2}$ ) In this context, QD nanostructures are expected to have great impact both on future optoelectronic devices, as well as on the investigation of quantum physics and information related phenomena. ${ }^{3-5)}$ Molecular beam epitaxy (MBE)-grown QDs are usually obtained with the StranskiKrastanov growth mechanism, in which the strain of a lattice mismatched layer is released through the formation of threedimensional islands. This type of growth results generally in dislocation-free high quality islands with a certain size distribution. ${ }^{6,7)}$ For future device applications and fundamental studies, controlling the assembly of QDs in a deterministic way is highly desirable.

Previous methods for controlling the size distribution and arrangement of $\operatorname{In}(\mathrm{Ga}) \mathrm{As} / \mathrm{GaAs}$ QDs have included growth on vicinal surfaces, on high index facets ${ }^{8-10)}$ and on patterned substrates. ${ }^{1-13)}$ In the cases where the QDs were optically active, the improvement in size control has resulted in emission spectra with significantly smaller line widths. ${ }^{14)}$ To date and to our knowledge, the smallest line width reported for an ensemble of InGaAs/GaAs QDs is around $10 \mathrm{meV} .{ }^{15)}$ This was achieved by very careful control of the synthesis conditions, so that the size distribution was minimized. Recently, we have demonstrated the synthesis of long-range ordered chains of InAs QDs by growth on a (110) cleaved facet composed of AlAs stripes embedded in GaAs. By controlling the position and size of the quantum dots using AlAs stripes, small size distributions could be achieved, leading to the question of whether this translates into an improvement in the optical properties of the InAs QDs. ${ }^{16,17)}$ In our initial work, ${ }^{16)}$ the QD arrays co-existed with microscopic triangular InAs islands. These defects arise from the heteroepitaxy of InAs on (110) GaAs surfaces and often crossed the QD arrays. In a later work, ${ }^{17)}$ a study of the growth mechanisms led to the fabrication of InAs QD arrays

*E-mail address: anna.fontcuberta-morral@epfl.ch without the appearance of these defects and in a reproducible way. The optical properties of these defect-free arrays are to date not characterized.

In this paper, we present scanning confocal photoluminescence (PL) spectroscopy measurements of defect-free InAs QD array ensembles, both as power and polarization dependent PL, and compare them to the QD morphology as analyzed by atomic force microscopy (AFM).

\section{Experimental Procedure}

\subsection{Growth}

The growth was carried out in a GenII MBE machine, by adapting the cleaved edge overgrowth (CEO) technique to the self assembly formation of InAs nanostructures. The main principle of the CEO technique is to grow on a surface that has been freshly cleaved in situ in the MBE machine. ${ }^{18)}$ The first MBE step consists of the preparation of the substrate by growing an alternating series of AlAs and GaAs layers on a (001) oriented GaAs surface. The cleaving along the [110] direction opens a new (110) surface, formed by AlAs and GaAs stripes. Immediately after the cleaving, InAs deposition begins and QD arrays form selectively on top of the AlAs layers.

The geometry of the freshly cleaved (110) surface strongly affects the growth of InAs, which can form different types of ordered nanostructures, ranging from nanowires to single and double QD chain arrays. We have previously shown that the occurrence of each of the structures depends on the thickness of the AlAs stripes and on the growth parameters. ${ }^{17)}$ Details of the growth process and technique have been presented elsewhere. ${ }^{17,19)}$ In the present work, we have restricted our attention to the study of single QD chains having different widths. These were grown on five AlAs/GaAs multistripe structures (MS) spatially separated by $1 \mu \mathrm{m}$ of GaAs. Each MS contains 10 AlAs stripes of thickness $d$, separated by GaAs interlayers $70 \mathrm{~nm}$ thick. $d$ is varied in subsequent MS from 20 to $40 \mathrm{~nm}$ in steps of $5 \mathrm{~nm}$. The growth conditions of the InAs nanostructures are as follows: In growth rate of $0.06 \AA / \mathrm{s}$, a nominal thickness of InAs of 1.5 monolayers (ML), a substrate temperature of $455^{\circ} \mathrm{C}$ and background $\mathrm{As}_{4}$ overpressure of $5.5 \times 10^{-5}$ mbar. $^{17)}$ The InAs deposition 

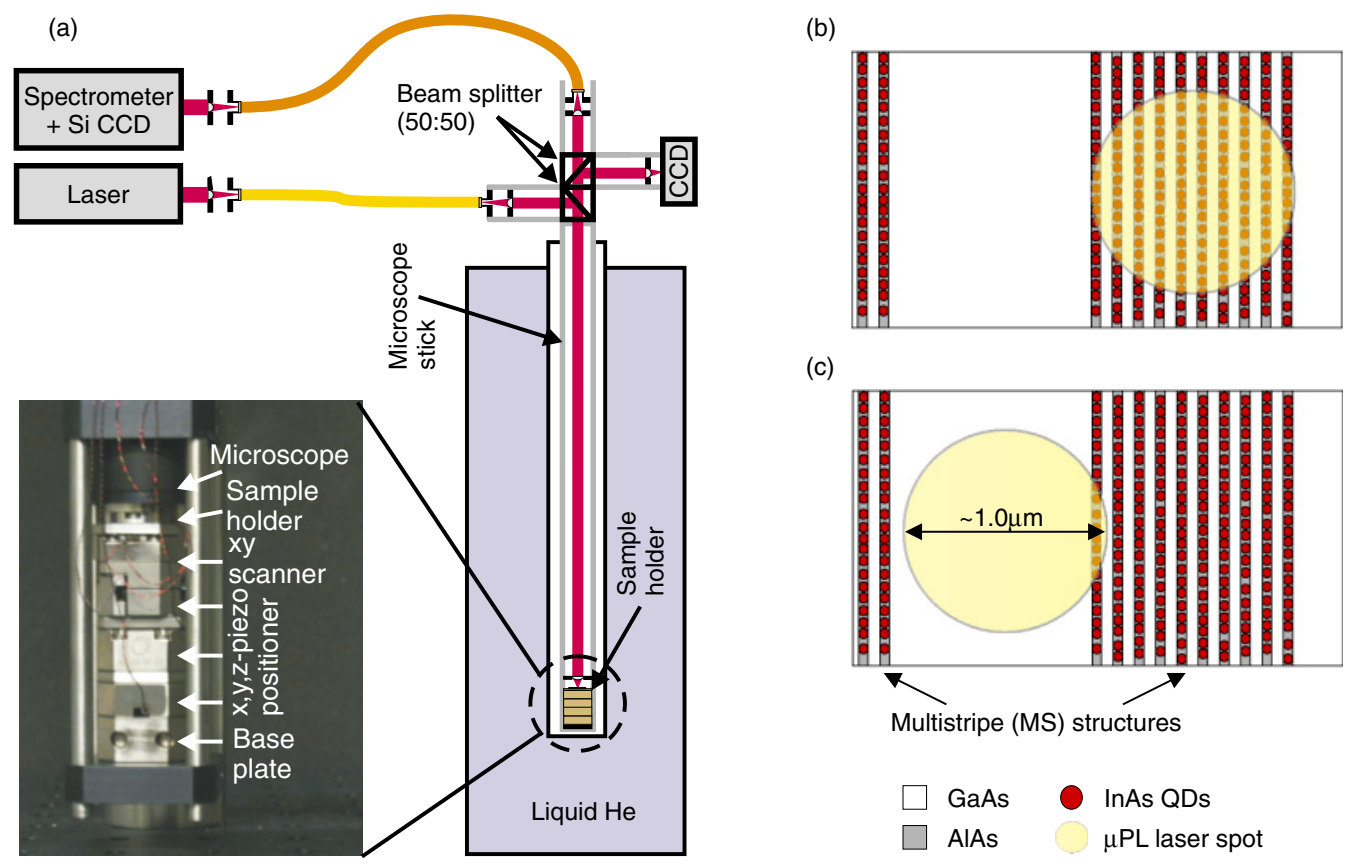

(c)

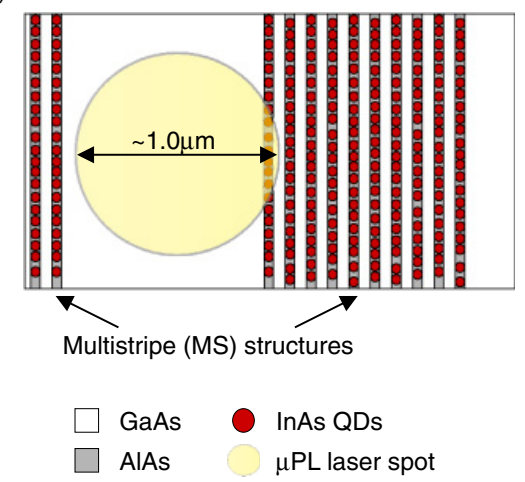

Fig. 1. (Color online) (a) Schematic of the system used for the $\mu \mathrm{PL}$ measurements. The inset shows a zoom-in of the sample holder that is located at the end of the microscope stick inside the liquid $\mathrm{He} .(\mathrm{b}, \mathrm{c})$ Considering the geometry of the sample and the resolution achieved in the $\mu \mathrm{PL}$ system, it is possible to excite either (b) QD ensembles or (c) few (single) QDs, depending on the microscope position with respect to the sample geometry.

is followed by $120 \mathrm{~s}$ of annealing at the same temperature under $\mathrm{As}_{4}$ overpressure. For the investigation of the morphology of the InAs nanostructures by AFM, the samples are cooled immediately without any further processing. For the study of the optical properties, the samples are capped with a $50 \mathrm{~nm}$ thick GaAs layer in order to avoid influence on the luminescence by the surface states and to ensure QD behavior (substrate temperature and $\mathrm{As}_{4}$ overpressure are the same as for the InAs deposition).

\subsection{Optical measurements}

The optical properties of the grown structures were investigated by using a commercially available scanning confocal $\mu \mathrm{PL}$ microscopy system (attoCFM I ${ }^{20)}$ ), having lateral displacement accuracy in the nanometer range. A schematic drawing of the setup is plotted in Fig. 1(a). The excitation and collection spot was slightly less than $1 \mu \mathrm{m}$ in diameter. The measurements were realized at $4.2 \mathrm{~K}$, using as excitation source either the $632.8 \mathrm{~nm}$ line of a $\mathrm{He}-\mathrm{Ne}$ laser or the $780 \mathrm{~nm}$ line of a laser diode. The luminescence from the sample was detected and analyzed by a combination of grating spectrometer and Si charge coupled device.

As will be described below, it is possible to obtain luminescence from QD ensembles as well as from only few or even single QDs. This can be achieved by either focusing the excitation spot at the center of the MS region, or by moving it away from the stripes such that only few QDs are contained within the collection area of the microscope. Figures 1(b) and 1(c) schematically represent how the spot of the confocal microscope should be placed on the sample for the investigation of either ensemble or few (single) QDs. A decrease in the QD PL intensity can help to accurately move the excitation spot from the center of the MS towards the edge. As a consequence of the reduction of the signal at the edge of the MS, longer time integration measurements are needed.

\section{Results and Discussion}

\subsection{Spatial dependence of the photoluminescence}

A typical PL intensity map corresponding to a one-dimentional (1D) scan on a large part of the sample is shown in Fig. 2(a). The scan includes both the substrate and MS regions. The $x$ - and $y$-axes correspond respectively to the energy scale and spatial coordinate of the scan across the stripes. The graph consists of a $15 \mu \mathrm{m}$ long scan taken with $0.25 \mu \mathrm{m}$ steps. The scan starts at the edge of the sample and moves towards the [001] direction. It contains the signal from five AlAs/GaAs MS as well as a section of the original GaAs substrate. A schematic drawing of the surface geometry is depicted to the left of the mapping scan for clarity. The dashed line indicates the interface between the epi-grown layers and the original substrate region. At the bottom of the scan, corresponding to the original GaAs substrate, we observe two notable features: (i) the GaAs free and shallow bound exciton peaks close to $1.51 \mathrm{eV}$ and (ii) the GaAs exciton peak bound to neutral (C) acceptor at $\sim 1.49 \mathrm{eV}^{21)}$ At the top of the scan, which corresponds to the region where the GaAs/AlAs MSs are present, the luminescence from the GaAs exciton is brighter than in the substrate. This could indicate that the epilayer contains fewer impurities, though the C-bound exciton is also slightly present. The free and shallow exciton lines located between 1.509 and $1.514 \mathrm{eV}$ are observed along the [001] direction. Interestingly, the intensity is modulated along the epilayer and appears to be brighter in the MS regions, as shown in Fig. 2(b). We believe that this is related to the lower effective refractive index of the AlAs/GaAs stack, ${ }^{22)}$ which ensures a better coupling with the confocal objective. The 


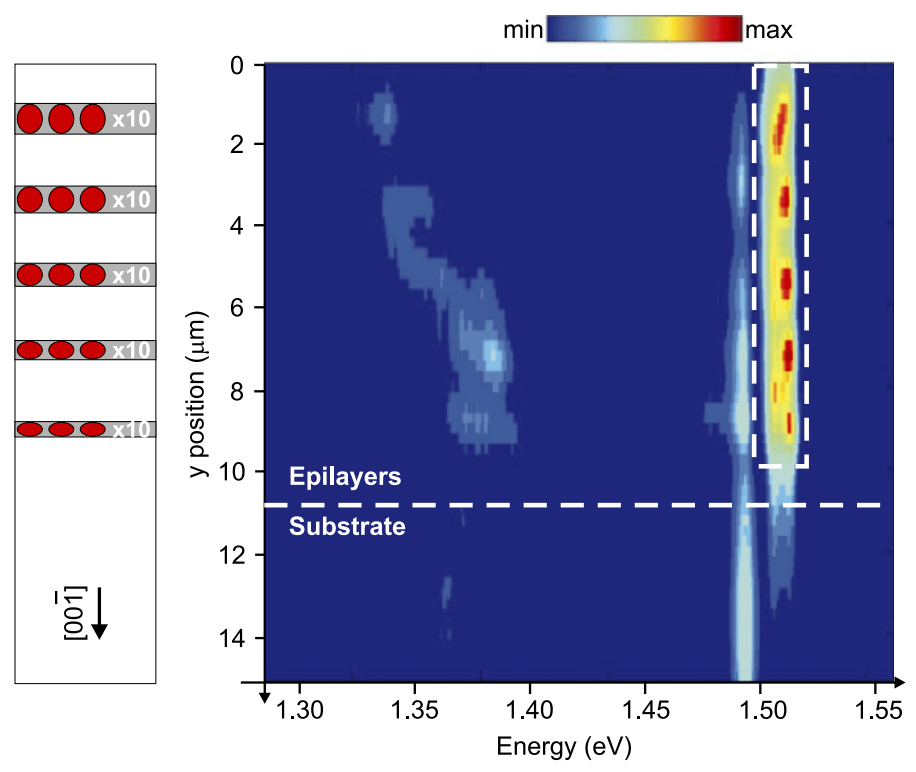

(a)

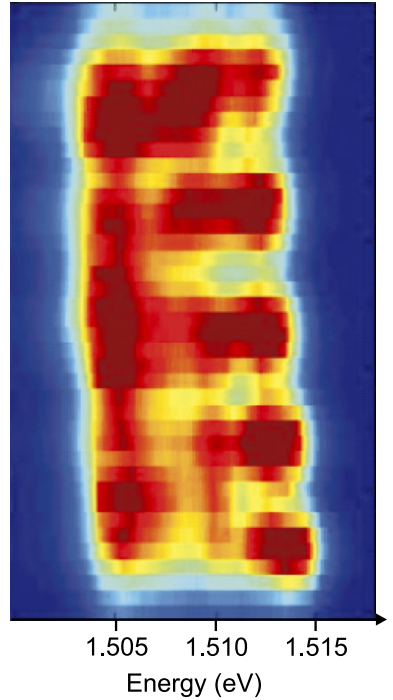

(b)

Fig. 2. (Color online) (a) 1D $\mu-\mathrm{PL}$ mapping scan, recorded at liquid He temperature, perpendicular to the AlAs-GaAs multistripe region. For $y=0-11 \mu \mathrm{m}$ the PL spectra are from the AIAs-GaAs multistripe regions, while larger $y$ values correspond to the substrate region (a schematic of the sample geometry is plotted to the left of the mapping scan). Color scale denotes normalized PL intensity, with dark blue being weak and red strongest. The nominal laser spot is less than $1.0 \mu \mathrm{m}$ in diameter with a power of $0.06 \mu \mathrm{W}$; integration time was 30 s per spectra; a dashed line has been added to mark the border between the substrate and the epilayers. (b) Zoom-in of the GaAs exciton energy region on the episide ( $y=0-10 \mu m)$, as indicated by a square area on (a). The GaAs exciton peak redshifts with increasing AIAs stripe thickness.

presence of tensile stress in the GaAs sandwiched between the multiple AlAs layers is also clear (the stress is due to a slight but non-zero difference in lattice constant). Indeed, we observe that the position of the free GaAs exciton peak shifts gradually to lower energies as the thickness of the AlAs layers is increased. ${ }^{19)}$

At energies well below the free and bound GaAs exciton peaks (between 1.30 and $1.40 \mathrm{eV}$ ), a second luminescence peak is observed. These peaks are broader (bands) and coincide with the location of the AlAs/GaAs multilayers. We attribute this luminescence to the InAs QDs obtained on the AlAs stripes. The center of the band shifts to lower energies along the [001] direction, at the same time that the AlAs stripes become broader (which means that the QDs are larger).

One should note that the intensity of the GaAs exciton peak is higher than that of the QDs. We believe that the following aspects can account for this: (i) the excitation energy is lower than the bandgap of the AlAs barrier layer below the QDs. For this reason, the transfer of electron-hole pairs from the surrounding material in the QDs is less efficient, compared with the standard case of InAs QDs grown on (001) oriented GaAs; (ii) there is a magnifying effect on the PL of the GaAs peak between the AlAs stripes. In this part of the structure the GaAs/AlAs multilayers are acting as mirrors. As consequence, the GaAs PL signal is not emitted in an isotropic way but is guided towards the surface of the sample. To validate this assumption, we note that the increased emission is only obtained between the AlAs stripes, while the PL signal of GaAs in the substrate far away from the MS regions is as intense as for the QDs.

Before analyzing in details the $\mu \mathrm{PL}$ spectra relative to the QD chains, it is necessary to look at the morphological characteristics of the uncapped sample. Typical AFM measurements of the uncapped sample are shown in Fig. 3(a). The MS structures can be identified by their characteristic formation of QD arrays on the AlAs stripes. It is important to note that the size of the QDs in the [001] direction, $d^{\prime}$, is determined directly by the thickness $d$ of the AlAs stripe underneath. Indeed, $d^{\prime}$ slightly exceeds the AlAs stripe thickness $d$ from $5 \%$ (40 nm thick stripe) up to $10 \%(20 \mathrm{~nm}$ AlAs stripes). ${ }^{23)}$ The fluctuation of the QDs lateral size is quite small, with FWHM from $4 \%$ on the largest AlAs stripes up to $7 \%$ on the thinnest ones. As presented in previous studies, ${ }^{17,25)}$ the height of the InAs QDs, $h$, is also proportional to the AlAs stripe thickness and the averaged values for each MS region are reported in Table I. The QDs present a height distribution of $\sim 15 \%$. This relation between QD height and AlAs stripe thickness is due to the significantly lower mobility and lifetime ${ }^{24)}$ of In adatoms in the (110) AlAs surface, with respect to (110) GaAs. ${ }^{25,26)}$ In agreement with the increase of $d$ in each MS structure, we have obtained five different QD ensembles, each one with a height distribution between 7.2 and $11.5 \mathrm{~nm}$, respectively, for MS widths from 20 to $40 \mathrm{~nm}^{27)}$ As shown in Fig. 3(b), the smaller stripes (MS20) host QDs with a narrower size distribution, and a standard deviation in height close to $2 \mathrm{~nm}$. For larger stripes, the distribution consistently broadens, attaining a standard deviation in height of $4 \mathrm{~nm}$ for the thickest AlAs stripes (MS40). From the considerations above and given that the QD height is much smaller than the width, we conclude that variations in height should mainly account for the change in confinement energy. The morphological characteristics for each of the QD ensembles are summarized in the Table I. Interestingly, the QD density is relatively constant as a function of $d$. 
(a)
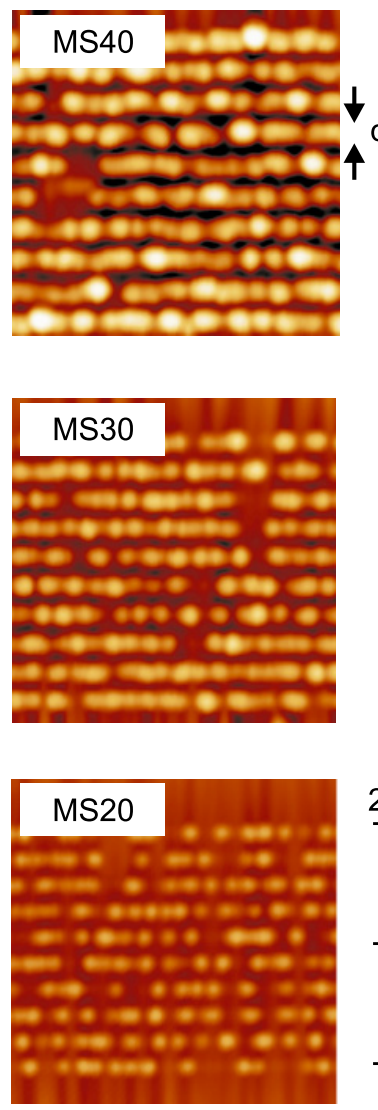

$23 \mathrm{~nm}$

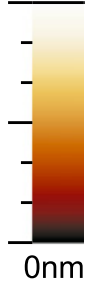

(b)

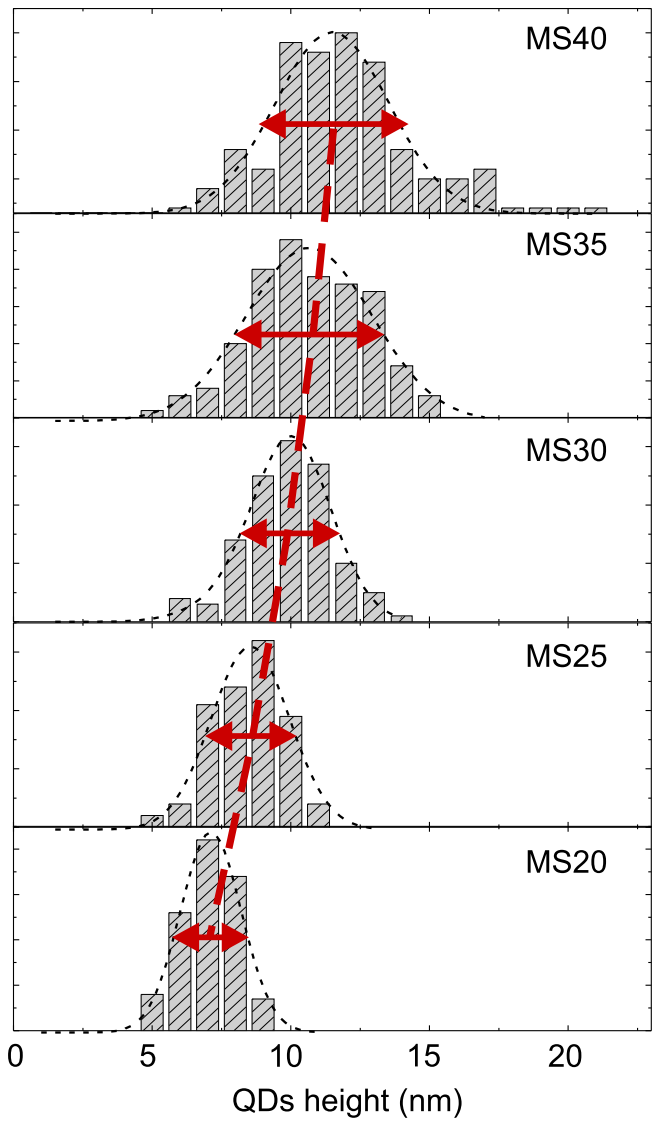

Fig. 3. (Color online) (a) AFM pictures of QD arrays grown on three different AIAs-aAs multistripes. From top to bottom, the AIAs thickness $d$ is 40 , 30 , and $20 \mathrm{~nm}$, while the GaAs thickness is always $70 \mathrm{~nm}$. Each scanned area is $1.1 \times 1.1 \mu \mathrm{m}^{2}$ broad, and the color bar is the same. (b) Height distribution representations of the InAs QDs, for different AIAs MS thicknesses. Red arrows denote standard deviation. From top to the bottom, $d$ decreases from 40 to $20 \mathrm{~nm}$ in steps of $5 \mathrm{~nm}$.

Table I. Properties of $1.5 \mathrm{ML}$ InAs QDs samples, without capping layer. $d$ represents the thickness of the AIAs stripes, $d^{\prime}$ is the QDs lateral size, $h$ is QDs height, $h: d^{\prime}$ is the aspect ratio in the growth direction and $\rho$ is the QD density. The density has been calculated on a $1 \mu \mathrm{m}^{2}$ area centered along the [001] direction with respect to each MS region. The GaAs regions between each AIAs stripe have been taken into account.

\begin{tabular}{lccccc}
\hline & $\begin{array}{c}\text { MS20 } \\
d=20 \mathrm{~nm}\end{array}$ & $\begin{array}{c}\text { MS25 } \\
d=25 \mathrm{~nm}\end{array}$ & $\begin{array}{c}\text { MS30 } \\
d=30 \mathrm{~nm}\end{array}$ & $\begin{array}{c}\text { MS35 } \\
d=35 \mathrm{~nm}\end{array}$ & $\begin{array}{c}\text { MS40 } \\
d=40 \mathrm{~nm}\end{array}$ \\
\hline$d^{\prime}(\mathrm{nm})$ & $29.0 \pm 3.8$ & $33.4 \pm 2.8$ & $36.5 \pm 2.2$ & $40.7 \pm 1.7$ & $42.8 \pm 1.7$ \\
$h(\mathrm{~nm})$ & $7.2 \pm 1.2$ & $8.5 \pm 1.4$ & $10.1 \pm 1.5$ & $10.7 \pm 2.3$ & $11.5 \pm 2.1$ \\
$h: d^{\prime}$ & 0.25 & 0.25 & 0.28 & 0.26 & 0.27 \\
$\rho\left(\mu \mathrm{m}^{-2}\right)$ & $126 \pm 6$ & $122 \pm 6$ & $130 \pm 7$ & $132 \pm 7$ & $121 \pm 6$ \\
\hline
\end{tabular}

Before presenting the results on the optical properties of the QD arrays, we would like to comment on the possible existence and/or influence of an InAs wetting layer (WL) below the QD chain, which would form a kind of ultrathin nanowire between the QDs. As shown in ref. 17, the precursors of the QD chains are thin nanowire structures that grow on top of the AlAs stripes. The height of these wires is smaller than the height of the dots, which implies larger quantization energy for the wire than for the QD chain. An excited electron-hole pair should preferentially relax into the quantized states of the dot and not of the WL. ${ }^{28)}$ This means that if this $1 \mathrm{D}$ wetting layer exists, the recombination would occur preferentially in the InAs QDs. In the case of InAs QDs grown on (110) AlAs surfaces, one would expect wetting layer PL emission in the $1.42-1.45 \mathrm{eV}$ energy range, as it was previously reported. ${ }^{29)}$ In our PL experiments we have never observed luminescence in this energy range, concluding that PL signal from InAs wetting layer does not exist.

Finally, we would like to point out the absence of InAs wetting layer PL signal from the GaAs region in-between the multistripes. Again, InAs QDs are not expected to form in (110) GaAs surfaces. However, one monolayer of InAs is expected to form. We have measured PL at $1.45 \mathrm{eV}$ only on the GaAs substrate, several microns away from the ML region. In agreement with this, AFM measurements on this part of the sample have indicated the presence of $1 \mathrm{ML}$ high InAs layer. The absence of the wetting layer between the MS regions is due to the migration of In adatoms towards the AlAs stripes, which lies at the origin of the formation of QDs only on the MS region. ${ }^{25)}$ Indeed, for the formation of QDs up to $11.5 \mathrm{~nm}$ high (40 ML), only 1.5 ML InAs needs to be deposited. There is indeed a redistribution of mass in the MS regions that hinders the formation of a wetting layer in the GaAs regions between the MS.

Figures 4(a) and 4(b) show PL mapping plots of the MS regions, whose spectra have been taken under two extremely different excitation powers and integration times, 0.008 and 

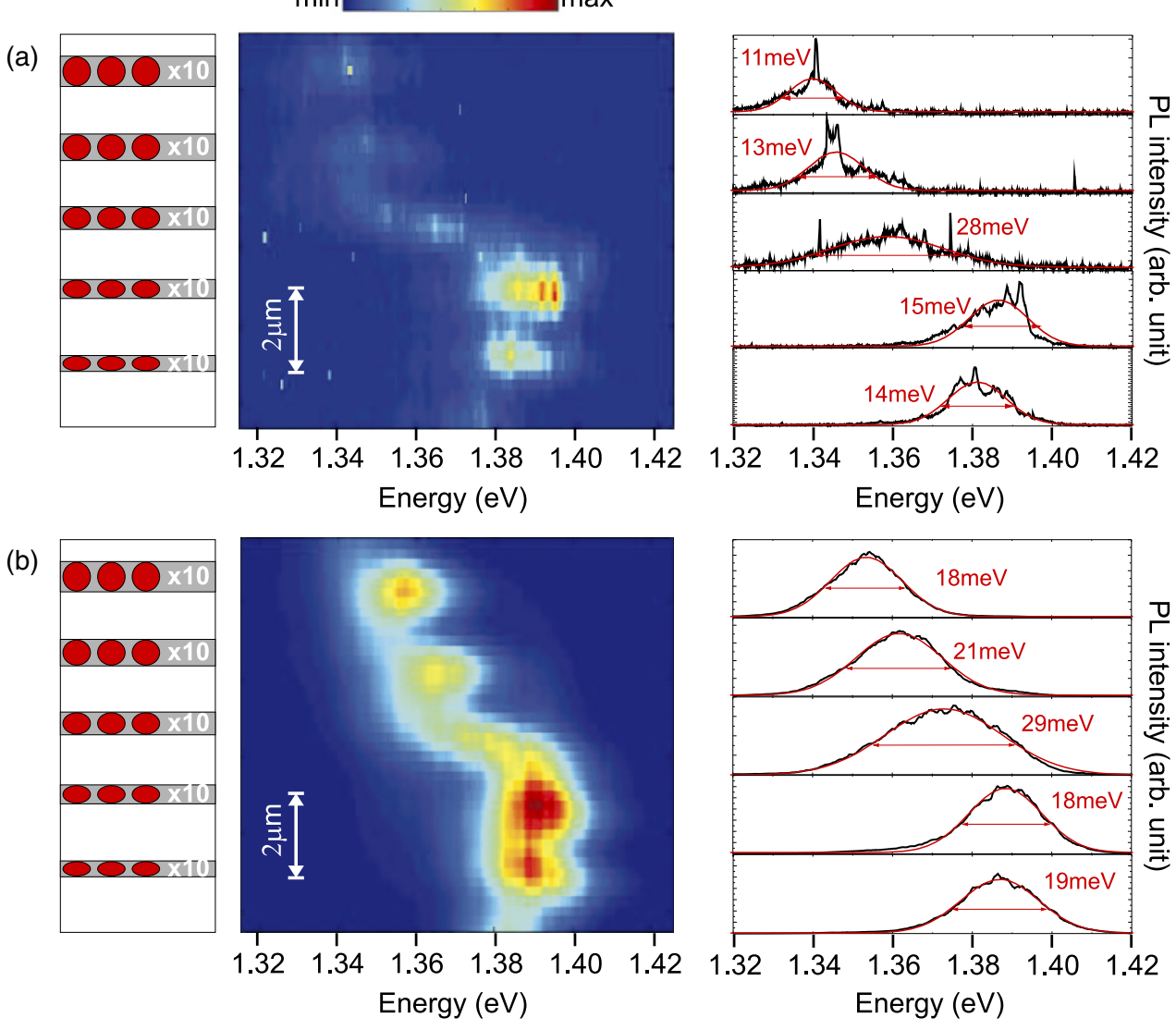

Fig. 4. (Color online) Power dependent $\mu$-PL mapping scans recorded from the same area of the AlAs-GaAs MS region, with the following measurement parameters: (a) low excitation power $(0.008 \mu \mathrm{W})$ and long integration time $(200 \mathrm{~s})$, (b) high excitation power $(5.6 \mu \mathrm{W})$ and short integration time $(5 \mathrm{~s})$. The spatial sampling is $0.25 \mu \mathrm{m}$. A schematic of the sample geometry is plotted to the left of each mapping scan, while integrated PL spectra from a spot on each multilayer region have been plotted to the right.

$5.6 \mu \mathrm{W}$ and respectively 200 and $5 \mathrm{~s}$. In the following, we will refer to these two measurements to low and high excitation power. We analyzed first the PL obtained under low excitation power conditions, shown in Fig. 4(a). The PL peak shifts from 1.340 to $1.385 \mathrm{eV}$ in the direction of decreasing stripe size (and QD size). For a better illustration of the shape and position of the spectra, a single line scan spectrum belonging to each MS region is plotted on the right side of Fig. 4(a). As it can be seen, the spectra are complex and formed by many sharp peaks. This can be attributed to the contribution of many QDs, even if the maximum number of QDs measurable at one time is 150. The shift of the energy peaks as a function of stripe size is consistent with the evolution of the QD dimension, as shown in Fig. 3(b). The PL peaks shift to lower energies for QDs grown on stripes from 25 to $40 \mathrm{~nm}$, and the QD heights increase from 8.5 to $11.5 \mathrm{~nm}$. Strikingly, very similar PL was measured for QDs with 25 and $20 \mathrm{~nm}$ widths. This is in principle in disagreement with the morphological analysis of the uncapped QDs which indicates a difference in size. We believe that this may be due to a partial change in the QD morphology after the deposition of a GaAs capping layer, as has been observed by several groups for the InAs-GaAs QDs system. ${ }^{30)}$

In order to understand the trend of the peak position with respect to QD size, the position of the emission lines can be modeled using the particle in a box approximation. ${ }^{31)}$ In our case, we assume for simplicity that (i) the QD base shape is symmetric (size $d^{\prime}$ determined by the AlAs stripe width $d$ ) and (ii) the QD height $h$ is proportional to $d$. Therefore, the QD energy level should be proportional to $1 / d^{2}$. In Fig. 5 we have plotted the peak energy position as function of the stripe thickness $d$ under different excitation power conditions. As expected, the energy peaks blueshift with decreasing $d$. The trend is respected from 40 to $25 \mathrm{~nm}$ stripe thickness in all the measurements, while a deviation from the trend appears at the $20 \mathrm{~nm}$ thick AlAs stripes. We believe this deviation to be due to a change in the morphology of the smallest QDs after capping with GaAs, from QD-like to wire-like. The capping process constitutes a slight erosion of the QD apex which reduces the QD height. This process affects to a higher degree the shape of small QDs than that of larger QDs. The appearance of nanowire-like InAs structures is possible in this scenario, changing the luminescence characteristics.

With the exception of the arrays obtained on MS30, the widths of the emission lines are relatively small, below $15 \mathrm{meV}$ at low excitation power; this is in agreement with a good size uniformity of the QDs. A similar trend is observed at high excitation powers. As expected, the line width increases with excitation power, and the sharp features also disappear, giving rise to a more homogeneous band. This 


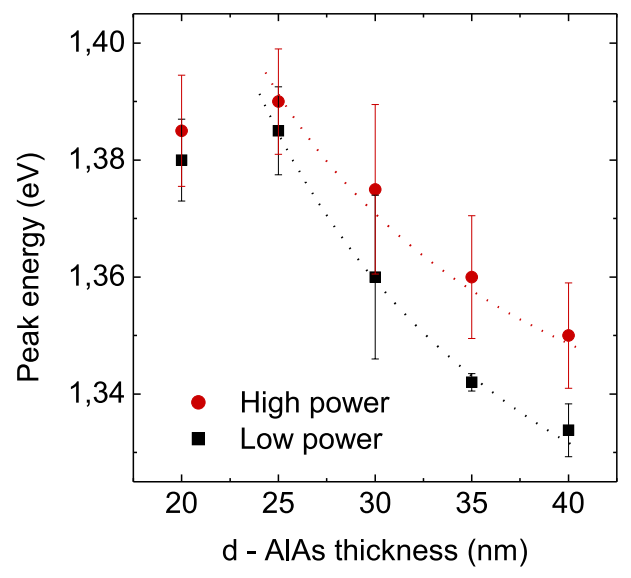

Fig. 5. (Color online) Peak energy position of PL versus stripe thickness $d$ for QDs ensemble on MS of thickness varying from 20 to $40 \mathrm{~nm}$ under different excitation powers (with reference to Fig. 4). The energy redshifts with increasing stripe thickness, except for the thinnest MS. At high excitation power the difference in energy is reduced between large and narrow AIAs stripes.

can be attributed to the existence of multiple exciton effects and to the recombination from higher excited levels, which occurs when the lower energy levels saturate. The linewidth of QDs from MS30 changes less significantly with excitation power compared to the linewidth of QDs from others MS regions. Furthermore, we observe that the highest PL intensity has been recorded on the thinner stripes (MS25, MS20). We believe that the observed weaker PL intensity from ensembles of larger QDs is related to an increment of strain accumulated in the apex, which can affect the radiative recombination processes. To understand this in more detail, more refined structural studies are needed.

\subsection{Detailed power dependent PL experiments on ensemble and single QDs}

In this section we present PL spectra recorded as a function of excitation power. We will show (i) evidence of shell filling in the spectroscopy of QD ensembles and (ii) the existence of single, double and multi exciton complexes in single QDs.

The detailed PL spectra of an ensemble of QDs grown on MS30 as a function of excitation power are shown in Fig. 6. The spectra are obtained with excitation powers in the range between 0.01 and $5 \mu \mathrm{W}$. Clearly, at low excitation powers only one band centered at $1.363 \mathrm{eV}$ and about $20 \mathrm{meV}$ broad is observed. No sharp peaks are present, meaning that the collected PL comes indeed from a QD ensemble. By increasing the excitation power, the PL intensity increases. At the same time, a second band shifted by about $\sim 30 \mathrm{meV}$ towards higher energies appears. As it is usually obtained in similar QD systems, the two bands can be respectively attributed to the $\mathrm{s}$ and $\mathrm{p}$ shell filling in QDs. ${ }^{32)}$ Additionally, we would like to point out that a very small feature above $1.41 \mathrm{eV}$ seems to appear at the highest excitation power. Unfortunately, due to limitation in the experimental setup it is not possible to realize any correlation of this peak with emission from a higher confined shell.

We have shown that the collective response of the emission corresponds to what it is expected of a quantum dot

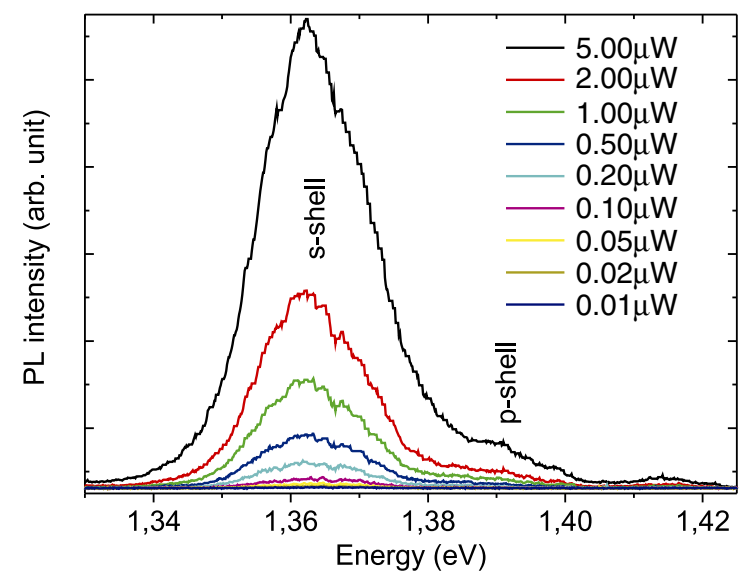

Fig. 6. (Color online) PL power series on QD ensemble from MS30. At low excitation power only a peak centered above $1.36 \mathrm{eV}$ dominates the spectra, while at large excitation power a second peak appears at larger energy $(1.39 \mathrm{eV})$. We attribute the two peaks to exciton recombination from $s$ and $p$ shell respectively in the QDs.

ensemble. Now, for consistency, we present the results on single QDs. An example of single QD luminescence nucleated on MS40 is shown in Fig. 7(a). The spectra have been recorded by excitation by a laser diode and for an integration time of $120 \mathrm{~s}$. At the lowest excitation power, only one peak is observed at $1.346 \mathrm{eV}$, which we attribute to single exciton $\left(\mathrm{X}^{0}\right)$. The FWHM of the peak is below $1 \mathrm{meV}$, at the limit of our spectrometer resolution. By increasing the excitation power, a sharp peak appears close to the $\mathrm{X}^{0}$ line, shifted to lower energies $(1.344 \mathrm{eV})$ and with a lower intensity. This peak corresponds to the bi-exciton (2X). By further increasing the excitation power, the intensity of both peaks increases. The intensity of the $2 \mathrm{X}$ line increases at nearly double the rate of the single exciton line. At the highest excitation power other peaks appear. These could be related either to the recombination of multiple excitons in the same dot or from vicinal $\operatorname{dot}(\mathrm{s})$, as the spot size tends to increase for the highest excitation powers. In Fig. 7(b) the integrated PL intensity versus the excitation power is presented in a log-log plot for the single exciton $\left(\mathrm{X}^{0}\right)$ and biexciton (2X) peaks. The fit reveals a quasi linear dependence between peak intensity and excitation power for the exciton and quasi quadratic for the biexciton. This is in agreement with what it is expected from a single and double exciton of InAs QDs. ${ }^{33,34)}$

\subsection{Polarization dependent photoluminescence}

In this section we present the polarization dependence of the PL measured in the InAs QD arrays. This study allows investigation of the anisotropy of the luminescence. In the following, parallel and perpendicular polarization will be referenced to the AlAs stripes. In Fig. 8(a) we show two spectra corresponding to parallel and perpendicular polarization of the PL taken with few QDs grown on the MS30. The spectra correspond respectively to the configurations exhibiting maximum and minimum PL intensity. The highest PL intensity is parallel to the [110] direction and the lowest is parallel to the [001] direction. All investigated QDs show the same trend. A schematic drawing of the setup configuration is shown in Fig. 8(b). 


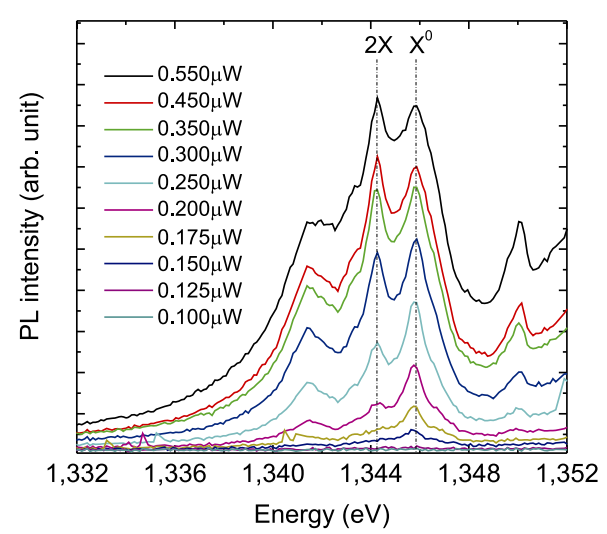

(a)

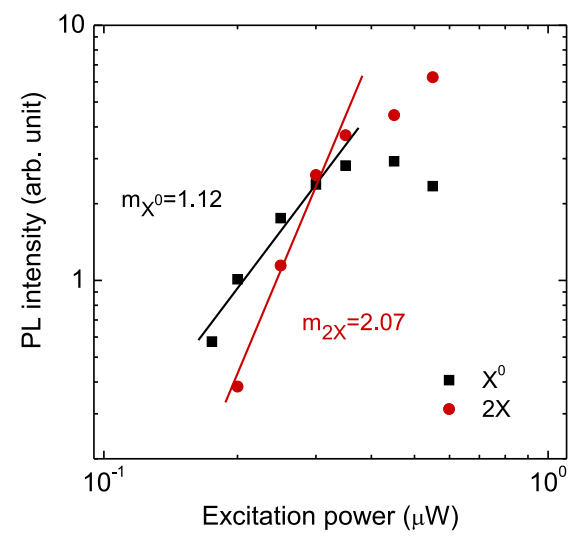

(b)

Fig. 7. (Color online) Few QDs PL spectroscopy from MS40. (a) PL power series spectra show appearance and evolution of different peaks depending of the excitation power. Two peaks dominate the spectra and we attribute these to single exciton ( $\left.\mathrm{X}^{0}\right)$ and biexciton (2X). (b) Logarithmic plot of the integrated PL intensity for exciton and biexciton lines versus the excitation power. The slopes of the relative trends in the linear regime correspond to the expected single exciton and biexciton lines behavior.

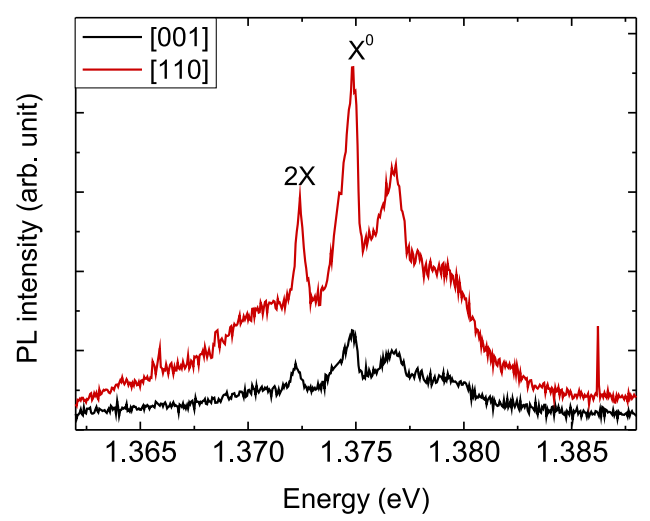

(a)

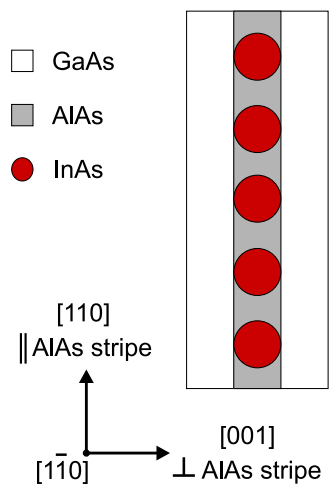

(b)

Fig. 8. (Color online) Polarization dependent PL from QDs on MS30. (a) PL spectra with the polarizer along the [001] and [110] directions. The QD luminescence clearly increases parallel to the stripe ([110] direction). Excitation power is $0.025 \mu \mathrm{W}$ and integration time $120 \mathrm{~s}$. (b) Schematics of the sample orientation with respect to the incident light, as reference for the polarization PL series orientation. The QD chains nucleate on the AIAs stripes and therefore are oriented parallel to the [110] direction.

Results of a full polarization PL series are reported in Fig. 9 in the form of a polar plot. The measurement series come from single QD spectroscopy on MS30 with polarization angle steps of $15^{\circ}$. The graph describes the behavior for single exciton $\left(\mathrm{X}^{0}\right)$ and biexciton $(2 \mathrm{X})$ lines. On the circular scale is reported the polarization angle and on the radial scale the PL intensity. Clearly, the maximum intensities are reached when the polarization analyzer is at $0^{\circ}$, meaning that the QDs PL intensity is polarized along the [110] direction, parallel to the AlAs stripes. The PL intensity changes with a period of $180^{\circ}$. Generally, the anisotropic polarization properties of QDs can be ascribed to the morphological in-plane anisotropy of the QDs. For example, asymmetry in the morphology of InAs/GaAs QDs has been reported to increase when the QDs are grown on high Miller index GaAs surfaces instead of on the (001) GaAs surface. Indeed, quantum dashes elongated and arrow-like QDs have been reported respectively on (211) $\mathrm{B}^{35)}$ and (311)B GaAs surfaces, ${ }^{36,37)}$ while QDs present both round domes and square-based symmetric pyramids ${ }^{38)}$ on (001) surfaces. In

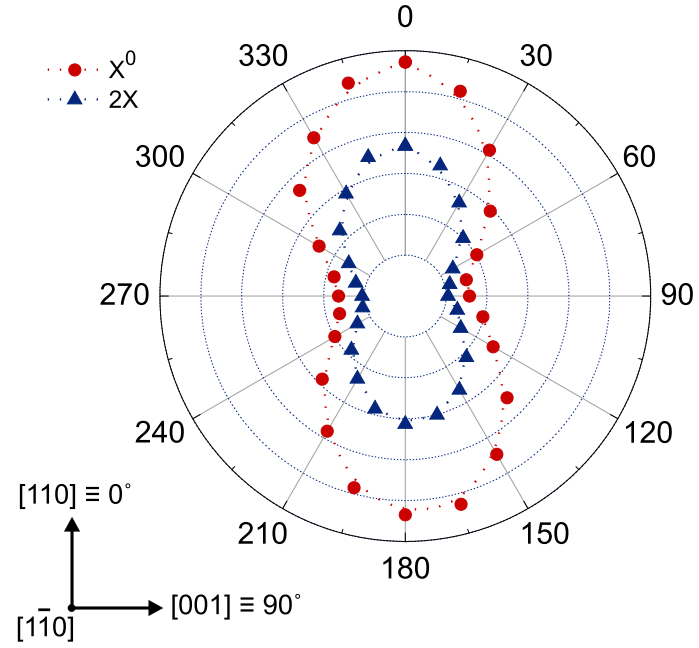

Fig. 9. (Color online) Polar plot for single exciton $\left(\mathrm{X}^{0}\right)$ and biexciton (2X) lines of a QD from MS30 (with reference to Fig. 8). QD PL intensity, represented in a radial scale, is highly polarized along $n \pi$ angles, which correspond to the [110] direction. Dot lines are depicted to guide the eye. 

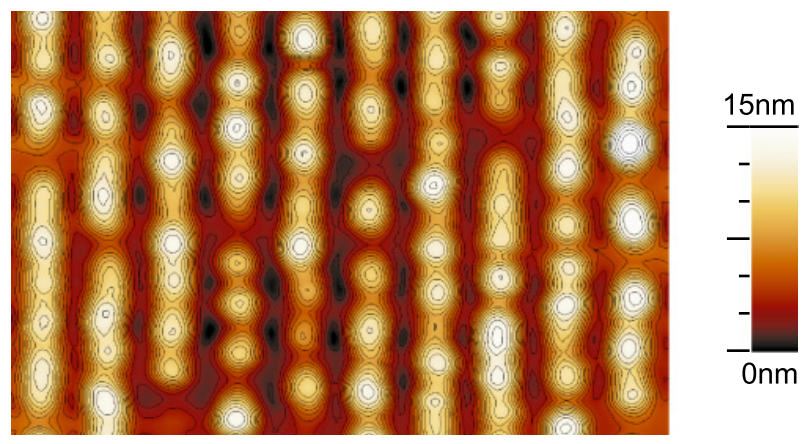

Fig. 10. (Color online) Contour plot AFM pictures on MS30 region for an uncapped sample. The difference in height between each contour line corresponds to $\sim 0.85 \mathrm{~nm}$. Clearly, QD sections present a sharper profile perpendicularly than parallel to the AlAs stripe, which indicates a preferential elongation of the dots along the stripe.

these cases, asymmetry in the luminescence polarization has also been observed. We have studied the morphology of the QDs grown on the stripes by high resolution AFM. We have found that the QDs nucleated on the AlAs stripes have asymmetric in-plane shapes with elliptical bases, where the long and short axes are respectively parallel and perpendicular to the stripe. An example of a high resolution AFM image for uncapped dots grown on MS30 is shown in Fig. 10, in which the QDs do not exhibit a circular base, but a preferential elongation along the underlying AlAs stripes. The contour plots highlight the different in-plane aspect ratios. In order to verify quantitatively, QD sections along the [110] and [001] directions have been taken for a large number of QDs. The average values for in plane shape aspect ratio $\rho_{\text {shape }}$ are reported in Table II for different MS and follow the formula

$$
\rho_{\text {shape }}=\frac{d_{110}^{\prime}-d_{001}^{\prime}}{d_{110}^{\prime}+d_{001}^{\prime}}
$$

where $d_{110}^{\prime}$ and $d_{001}^{\prime}$ are the QD lateral size along and perpendicular to the AlAs stripe. To compare the morphological and optical anisotropy, the PL polarization ratio $\rho_{\text {optical }}$ has been also calculated for QDs from different MS regions. The data are reported in the Table II, where $\rho_{\text {optical }}$ is the fraction of polarized light along the stripe, given by

$$
\rho_{\text {optical }}=\frac{I_{110}-I_{001}}{I_{110}+I_{001}}
$$

where $I_{110}$ and $I_{001}$ are the integrated PL intensities along the two orthogonal [110] and [001] directions. Both morphological and optical data are in strong agreement. The QDs are elongated along the [110] direction and the PL exhibits higher intensity for this polarization direction. The values $\rho_{\text {shape }}$ and $\rho_{\text {optical }}$ are similar, which may be coincidence. The origin is still not clear and should be further investigated.

Finally, we compare our results on PL anisotropy from InAs QDs with the literature. Several groups have reported a net optical anisotropy of a few percent. ${ }^{35-37)}$ Only in ref. 39 a value of $30 \%$ (in the same range of polarization ratio measured in this work) has been reported. As shown in the Table II, we have also measured optical
Table II. Anisotropic properties of QDs grown on stripes from 20 to $40 \mathrm{~nm}$. $\rho_{\text {shape }}$ is the aspect ratio of the in-plane QD dimensions obtained by AFM investigations of uncapped samples. $\rho_{\text {optical }}$ is the polarization ratio obtained by polarization PL series. Both ratios indicate that along [110] there is respectively a "preferential" elongation in the shape and excitonic optical recombination. Additionally, $\rho_{\text {optical }}^{*}$ indicates the polarization ratio on a single QD.

\begin{tabular}{lccccc}
\hline & MS20 & MS25 & MS30 & MS35 & $\begin{array}{c}\text { MS40 } \\
d=20 \mathrm{~nm}\end{array}$ \\
& $d=25 \mathrm{~nm}$ & $d=30 \mathrm{~nm}$ & $d=35 \mathrm{~nm}$ & $d=40 \mathrm{~nm}$ \\
\hline$\rho_{\text {shape }}$ & $0.18 \pm 0.06$ & $0.14 \pm 0.05$ & $0.23 \pm 0.06$ & $0.24 \pm 0.04$ & $0.24 \pm 0.04$ \\
$\rho_{\text {optical }}$ & $0.25 \pm 0.01$ & $0.29 \pm 0.01$ & $0.29 \pm 0.01$ & $0.28 \pm 0.01$ & $0.35 \pm 0.02$ \\
$\rho_{\text {optical }}^{*}$ & - & - & 0.61 & - & 0.41 \\
\hline
\end{tabular}

anisotropy in a single dot $\left(\rho_{\text {optical }}^{*}\right)$ that reaches an extremely high polarization ratio up to $60 \%$. To our knowledge, only Favero et al. ${ }^{40)}$ have reported a larger polarization ratio (up to $82 \%$ ) for the case of a single InAs QD, by measuring very dilute QD samples. They observed that the polarization ratio decreases with QD density. Differently from them, our polarization dependent results have been obtained on single QD in a highly dense QD array. Therefore, we conclude that the optical anisotropy results presented here shows an exceptionally high polarization ratio for both QD ensembles $(\sim 30 \%)$ and a single QD ( $60 \%)$.

\section{Conclusions}

In conclusion, we have presented the characterization of optical properties for InAs QD arrays that were grown on the cleaved facet of a series of $\mathrm{AlAs} / \mathrm{GaAs}$ multilayer structures. The InAs QDs were defect-free, meaning that there were no InAs trapezoidal islands precipitated between the QDs. The width of the investigated arrays, which is determined by the surface geometry, was varied between 20 and $40 \mathrm{~nm}$. The PL emission lines redshift with increasing size of the dots, in agreement with theory. Significantly narrow emission lines are measured, in accordance with a narrow dot size distribution. Moreover, shell filling and measurements on single dots corroborate that the observed luminescence is originated at the QDs and not on the 1D wetting layer underneath. Finally, polarization dependence investigations on CEO defined InAs QDs have revealed anisotropic behavior of the emitted PL, with highest intensity parallel to the AlAs stripes. Careful AFM investigations of the in-plane QD shape showed a strong anisotropic elongation of the dots in the same geometry, which to a good extent explains the anisotropic behavior of the measured PL. These results further confirm the promising potential for CEO InAs QD arrays for new applications, in particular for polarization-sensitive devices.

\section{Acknowledgments}

This work was financially supported by the Deutsche Forschungsgemeinschaft (DFG) in the framework of SFB 631 TP B1 and the Nanosystems Initiative Munich (NIM), by the BmBF through the Grant NanoQUIT-01BM469, and by the Marie Curie Excellence Grant "SENFED". The authors kindly thank J. J. Finley for helpful discussions, M. Frimmer, D. Spirkoska, M. Heiß, and M. Heigoldt for technical assistance. 
1) D. Bimberg, M. Grundmann, and N. N. Ledentsov: Quantum Dot Heterostructures (Wiley, New York, 1998).

2) M. Grundmann: Nano-Optoelectronics: Concepts, Physics and Devices (Springer, Heidelberg, 2001).

3) K. H. Schmidt, G. Medeiros-Ribeiro, M. Oestreich, P. M. Petroff, and G. H. Dohler: Phys. Rev. B 54 (1996) 11346.

4) R. Heitz, M. Veit, N. N. Ledentsov, A. Hoffmann, D. Bimberg, V. M. Ustinov, P. S. Kop'ev, and Zh. I. Alferov: Phys. Rev. B 56 (1997) 10435

5) H. Shoji, K. Mukai, N. Ohtsuka, M. Sugawara, T. Uchida, and H. Ishikawa: IEEE Photonics Technol. Lett. 7 (1995) 1385.

6) H. Kissel, U. Müller, C. Walther, W. T. Masselink, Yu. I. Mazur, G. G. Tarasov, and M. P. Lisitsa: Phys. Rev. B 62 (2000) 7213.

7) L. Chu, M. Arzberger, G. Boehm, and G. Abstreiter: J. Appl. Phys. 85 (1999) 2355.

8) Y. Akiyama and H. Sakaki: Appl. Phys. Lett. 89 (2006) 183108.

9) M. Schmidbauer, Sh. Seydmohamadi, D. Grigoriev, Z. M. Wang, Yu. I. Mazur, P. Schaefer, M. Hanke, R. Koehler, and G. J. Salamo: Phys. Rev. Lett. 96 (2006) 066108.

10) R. Noetzel, Z. Niu, M. Ramsteiner, H.-P. Schoenherr, A. Tranpert, L. Daeweritz, and K. H. Ploog: Nature 392 (1998) 56.

11) D. Grützmacher, T. Fromherz, C. Dais, J. Stangl, E. Müller, Y. Ekinci, H. H. Solak, H. Sigg, R. T. Lechner, E. Wintersberger, S. Birner, V. Holý, and G. Bauer: Nano Lett. 7 (2007) 3150.

12) J. H. Lee, Z. M. Wang, W. T. Black, V. P. Kunets, Y. I. Mazur, and G. J. Salamo: Adv. Funct. Mater. 17 (2007) 3187.

13) R. Songmuang, S. Kiravittaya, and O. G. Schmidt: Appl. Phys. Lett. 82 (2003) 2892.

14) Q. Xie, N. P. Kobayashi, T. R. Ramachandran, A. Kalburge, P. Chen, and A. Madhukar: J. Vac. Sci. Technol. B 14 (1996) 2203.

15) Z. Mi and P. Bhattacharya: J. Appl. Phys. 98 (2005) 023510.

16) J. Bauer, D. Schuh, E. Uccelli, R. Schulz, A. Kress, F. Hofbauer, J. J. Finley, and G. Abstreiter: Appl. Phys. Lett. 85 (2004) 4750.

17) E. Uccelli, M. Bichler, S. Nürnberger, G. Abstreiter, and A. Fontcuberta i Morral: Nanotechnology 19 (2008) 045303.

18) L. Pfeiffer, K. W. West, H. L. Stormer, J. P. Eisenstein, K. W. Baldwin, D. Gershoni, and J. Spector: Appl. Phys. Lett. 56 (1990) 1697.

19) E. Uccelli, J. Bauer, M. Bichler, D. Schuh, J. J. Finley, G. Abstreiter, and A. Fontcuberta i Morral: Self-Assembled Quantum Dots (Springer, Heidelberg, 2008) p. 25.

20) http://www.attocube.com

21) L. Pavesi and M. Guzzi: J. Appl. Phys. 75 (1994) 4779.

22) Refractive indices for GaAs and AlAs in the investigated energy range are respectively equal to $n_{\mathrm{GaAs}} \sim 4$ and $n_{\mathrm{AlAs}} \sim 3$ [N. Pikhtin and A. D. Yas'kov: Sov. Phys. Semicond. 14 (1980) 389].

23) As is common in AFM analysis of self assembled QDs, an overestimation of the actual QD sizes has to be taken into account due to oxidization of the surface as well as the convolution of the sample's surface morphology with the shape of the AFM tip. In particular, the overestimation usually affects the QD width $\left(d^{\prime}\right)$ more than the QD height $(h)$. Here, we comment that the data for $d^{\prime}$ follow the actual trend inside the intrinsic limit of the AFM tip dimension, as reported in Table I, where the difference between $d$ and $d^{\prime}$ increases with decreasing $d$.

24) Adatom lifetime is the time between the atom impingement on the surface and the following nucleation or desorption process.

25) E. Uccelli, S. Nürnberger, M. Bichler, G. Abstreiter, and A. Fontcuberta i Morral: Superlattices Microstruct. 44 (2008) 425.

26) X. B. Niu, E. Uccelli, A. Fontcuberta i Morral, and C. Ratsch: Appl. Phys. Lett. 95 (2009) 023119.

27) We would like to note here that the absolute height differs by approximately $15 \%$ from our previous publication (ref. 17). This is due to the fact that the AFM measurements were realized with a new instrument, having much better accuracy.

28) M. Grundmann, N. N. Ledentsov, O. Stier, J. Böhrer, D. Bimberg, V. M. Ustinov, P. S. Kop'ev, and Zh. I. Alferov: Phys. Rev. B 53 (1996) R10509.

29) M. Hadjipanayi, A. C. Maciel, J. F. Ryan, D. Wasserman, and S. A. Lyon: Appl. Phys. Lett. 85 (2004) 2535.

30) F. Ding, L. Wang, S. Kiravittaya, E. Müller, A. Rastelli, and O. G. Schmidt: Appl. Phys. Lett. 90 (2007) 173104.

31) C. Schüller: Inelastic Light Scattering of Semiconductors Nanostructures-Fundamentals and Recent Advances (Springer, Heidelberg, 2006).

32) P. Hawrylak, G. A. Narvaez, M. Bayer, and A. Forchel: Phys. Rev. Lett. 85 (2000) 389.

33) F. Findeis, A. Zrenner, G. Böhm, and G. Abstreiter: Solid State Commun. 114 (2000) 227

34) J. J. Finley, A. D. Ashmore, A. Lemaitre, D. J. Mowbray, M. S. Skolnick, I. E. Itskevich, P. A. Maksym, M. Hopkinson, and T. F. Krauss: Phys. Rev. B 63 (2001) 073307.

35) S. P. Guo, H. Ohno, A. Shen, F. Matsukura, and Y. Ohno: Appl. Phys. Lett. 70 (1997) 2738

36) M. Henini, S. Sanguinetti, S. C. Fortina, E. Grilli, M. Guzzi, G. Panzarini, L. C. Andreani, M. D. Upward, P. Moriarty, P. H. Beton, and L. Eaves: Phys. Rev. B 57 (1998) R6815.

37) S. Sanguinetti, S. Castiglioni, E. Grilli, M. Guzzi, G. Panzarini, L. C. Andreani, and M. Henini: Jpn. J. Appl. Phys. 38 (1999) 4676.

38) P. Kratzer, Q. K. K. Liu, P. Acosta-Diaz, C. Manzano, G. Costantini, R. Songmuang, A. Rastelli, O. G. Schmidt, and K. Kern: Phys. Rev. B 73 (2006) 205347.

39) C. Santori, D. Fattal, M. Pelton, G. S. Solomon, and Y. Yamamoto: Phys. Rev. B 66 (2002) 045308.

40) I. Favero, G. Cassabois, A. Jankovic, R. Ferreira, D. Darson, C. Voisin, C. Delalande, Ph. Roussignol, A. Badolato, P. M. Petroff, and J. M. Gérard: Appl. Phys. Lett. 86 (2005) 041904. 